\title{
COVID-19 incidence and mortality in Nigeria: Gender based analysis
}

\author{
Olubukola O Olusola-Makinde ${ }^{\text {Corresp., } 1}$, Olusola S Makinde ${ }^{2}$ \\ ${ }^{1}$ Department of Microbiology, Federal University of Technology, Akure, Akure, Ondo, Nigeria \\ 2 Department of Statistics, Federal University of Technology, Akure, Akure, Ondo, Nigeria \\ Corresponding Author: Olubukola O Olusola-Makinde \\ Email address: ooolusola-makinde@futa.edu.ng
}

Background: Coronavirus Disease 2019 (COVID-19) has been surging globally. Risk strata in medical attention are of dynamic significance for apposite assessment and supply distribution. Presently, no known cultured contrivance is available to fill this gap of this pandemic. The aim of this study is to develop a predictive model based on vector autoregressive moving average (VARMA) model of various orders for gender based daily COVID-19 incidence in Nigeria. This study also aims to proffer empirical evidence that compares incidence between male and female for COVID-19 risk factors.

Methods: Wilcoxon signed-rank test is employed to investigate the significance of the difference in the gender distributions of the daily incidence. A VARMA model of various orders is formulated for the gender based daily COVID-19 incidence in Nigeria. The optimal VARMA model is identified using Bayesian information criterion. Also, a predictive model based on univariate autoregressive moving average model is formulated for the daily death cases in Nigeria. Fold change is estimated based on crude case-fatality risk to investigate whether there is massive underreporting and under-testing of COVID-19 cases in Nigeria.

Results: Daily incidence is higher in males on most days from April 11, 2020 to September 12, 2020. Result of Wilcoxon signed-rank test shows that incidence among male is significantly higher than female $\left(p\right.$-value $\left.<2.22 \times 10^{-16}\right)$. White neural network test shows that daily female incidence is not linear in mean $(p$-value $=0.00058746)$ while daily male incidence is linear in mean ( $p$-value $=0.4257)$. McLeod-Li test shows that there is autoregressive conditional heteroscedasticity in the female incidence (Maximum $\mathrm{p}$ value $=1.4277 \times 10^{-5}$ ) and male incidence (Maximum $p$-value $=9.089 .0816 \times 10^{-14}$ ) at $5 \%$ level of significance. Ljung-Box test (Tsay, 2014) shows that the daily incidence cases are not random ( $p$ value $=0.0000$ ). The optimal VARMA model for male and female daily incidence is $\operatorname{VARMA}(0,1)$. The optimal model for the Nigeria's daily COVID-19 death cases is identified to be $\operatorname{ARIMA}(0,1,1)$. There is no evidence of massive underreporting and under-testing of COVID-19 cases in Nigeria.

Conclusions: Comparison of the observed incidence with fitted data by gender shows that the optimal VARMA and ARIMA models fit the data well. Findings highlight the significant roles of gender on daily COVID-19 incidence in Nigeria. 


\section{COVID-19 incidence and mortality in Nigeria: Gender} 3 based analysis

4

5

Olubukola Olayemi Olusola-Makinde ${ }^{1}$, Olusola Samuel Makinde ${ }^{2}$

${ }^{1}$ Department of Microbiology, Federal University of Technology, P.M.B 704, Akure, Ondo State, Nigeria

${ }^{2}$ Department of Statistics, Federal University of Technology, P.M.B 704, Akure, Ondo State, Nigeria

Corresponding Author:

Olubukola Olusola-Makinde ${ }^{1}$

Department of Microbiology, Federal University of Technology, P.M.B. 704, Akure, Ondo State, Nigeria

Email address: ooolusola-makinde@futa.edu.ng

\section{Abstract}

Background: Coronavirus Disease 2019 (COVID-19) has been surging globally. Risk strata in medical attention are of dynamic significance for apposite assessment and supply distribution. Presently, no known cultured contrivance is available to fill this gap of this pandemic. The aim of this study is to develop a predictive model based on vector autoregressive moving average (VARMA) model of various orders for gender based daily COVID-19 incidence in Nigeria. This study also aims to proffer empirical evidence that compares incidence between male and female for COVID-19 risk factors.

Methods: Wilcoxon signed-rank test is employed to investigate the significance of the difference in the gender distributions of the daily incidence. A VARMA model of various orders is formulated for the gender based daily COVID-19 incidence in Nigeria. The optimal VARMA model is identified using Bayesian information criterion. Also, a predictive model based on univariate autoregressive moving average model is formulated for the daily death cases in Nigeria. Fold change is estimated based on crude case-fatality risk to investigate whether there is massive underreporting and under-testing of COVID-19 cases in Nigeria.

Results: Daily incidence is higher in males on most days from April 11, 2020 to September 12, 2020. Result of Wilcoxon signed-rank test shows that incidence among male is significantly higher than female $\left(p\right.$-value $<2.22 \times 10^{-16}$ ). White neural network test shows that daily female incidence is not linear in mean ( $\mathrm{p}$-value $=0.00058746$ ) while daily male incidence is linear in mean ( $\mathrm{p}$-value $=0.4257$ ). McLeod-Li test shows that there is autoregressive conditional heteroscedasticity in the female incidence (Maximum p-value $=1.4277 \times 10^{-5}$ ) and male 
40 incidence (Maximum p-value $=9.0816 \times 10^{-14}$ ) at $5 \%$ level of significance. Ljung-Box test

41 (Tsay, 2014) shows that the daily incidence cases are not random ( $p$-value $=0.0000)$. The optimal

42 VARMA model for male and female daily incidence is $\operatorname{VARMA}(0,1)$. The optimal model for the

43 Nigeria's daily COVID-19 death cases is identified to be $\operatorname{ARIMA}(0,1,1)$. There is no evidence

44 of massive underreporting and under-testing of COVID-19 cases in Nigeria

45 Conclusions: Comparison of the observed incidence with fitted data by gender shows that the

46 optimal VARMA and ARIMA models fit the data well. Findings highlight the significant roles

47 of gender on daily COVID-19 incidence in Nigeria.

\section{Introduction}

49 The end of 2019 saw the rise of a new disease which originated from Wuhan metropolis in

50 China. World Health Organization (WHO) named the disease; novel coronavirus disease 2019

51 (COVID-19) and, by March 2020, it had become a pandemic (Purcell and Charles, 2020; Sohrabi

52 et al., 2020). The responsible virus is called severe acute respiratory syndrome coronavirus 2

53 (SARS-CoV-2) (Zhu et al., 2020; Gorbalenya et al., 2020), which shares about 80\% genetic

54 similarity with Middle East respiratory syndrome coronavirus (MERS-CoV) and the severe acute

55 respiratory syndrome coronavirus (SARS-CoV) (Drosten et al., 2020).

56 Some patients of this novel disease, COVID-19, develop moderate and life-threatening

57 conditions with symptoms such as acute respiratory distress syndrome (ARDS), acute respiratory

58 failure, coagulopathy, septic shock, and metabolic acidosis (Jin et al., 2020). Due to potentiality

59 of this disease to be severe and fatal, there is need to identify its risk factors especially for life-

60 threatening conditions. This is to ascertain the specific medical and demographic physiognomies

61 with more accuracy; it will equally enable the apposite supportive hands and rapid intensive care

62 unit (ICU) treatment when required.

63 In United States of America, as at August 7, 2020, the total number of confirmed cases was

$643,404,410$ and 118,145 mortality cases. Of the 118,145 deceased patients, 53\% were males,

65 while some other countries such as Peru, India, Burkina Faso, Guatemala, Kenya, Pakistan,

66 Afghanistan, Thailand, Bangladesh and Nepal had mortality rates of males as high as 70\%, 73\%,

$6774 \%, 74 \%, 74 \%, 74 \%, 76 \%, 76 \%, 77 \%$ and 81\% respectively (UNWOMEN, 2020). Even

68 though these data may be underreported, Lau et al (2020) employed mortality rate as yardstick

69 for data reliability. Meanwhile, more disparity in gender distribution of COVID-19 incidence has

70 been documented. Sohrabi et al. (2020), Del Rio and Malani (2020) and Cascella et al. (2020)

71 reported that severe manifestations are more common in males, particularly in the elderly.

72 Nigeria confirmed its index case in Lagos State on February 27, 2020 (NCDC, 2020a).

73 Thereafter, the first thirty-two (32) cases were managed in Mainland Hospital, Yaba, Lagos

74 State. Out of the 32 cases, 66\% were reported to be males (Bowale et al., 2020). As of

75 September 6, 2020, the total number of confirmed cases had risen to 54,905 with $64 \%$ being

76 males and 36\% females (NCDC, 2020b). This raises the question if it is a COVID-19 risk factor

77 to be male?

78 With focus on Nigeria data, this study considers daily incidence of COVID-19 reported cases

79 based on gender. It answers the question whether daily COVID-19 incidence in Nigeria is 
80

81

82

83

84

85

86

87

88

89

90

91

92

93

94

95

96

97

98

99

100

101

102

103

104

105

106

107

108

109

110

111

112

113

114

115

116

117

118

119

gender-skewed, as well as provides relationship between daily incidence and gender. A predictive model based on vector autoregressive moving average model is formulated for the gender based daily incidence in Nigeria. Similarly, a predictive model based on univariate autoregressive integrated moving average model is formulated for the daily death cases in Nigeria. The study targets the optimal fitting model for predictive and inferential purposes. Recent literature showed the relationship between countries' population and daily incidence cases among some African countries (Makinde et al., 2020b). The study also highlighted significant monotone trends in the daily COVID-19 incidence and mortality counts of many countries in Africa up to April 25, 2020. Makinde et al. (2020a) highlighted effect of modes of transmission of COVID-19 on prevalence and mortality counts in World Health Organisation (WHO) regions. Ojokoh et al. (2020) considered the impact of COVID-19 and lockdown policies on farming, food security and agribusiness in some West African countries. Some predictive models have been suggested in literature for modelling COVID-19 dataset. These include use of autoregressive integrated moving average (ARIMA) model (Benvenuto et al., 2020). Abdulmajeed et al. (2020) considered an ensemble model based on ARIMA model, a Holt-Winters exponential smoothing model and generalized autoregressive conditional heteroscedasticity model for incidence cases in Nigeria.

\section{Materials \& Methods}

\section{Data}

The Nigeria's COVID-19 incidence data by gender reported in this study have been sourced from the Nigeria Center for Disease Control (NCDC). The data is available at https://www.ncdc.gov.ng/diseases/sitreps. The data include Nigeria's daily COVID-19 incidence broken down by sex and daily death cases. Figure 1 presents plot of Nigeria's daily COVID-19 incidence by gender from April 11, 2020 to September 12, 2020. It is observed from the figure that the daily incidence is higher in males in most of the days under study. Daily total incidence was on the rise until the end of July. Figure 2 presents plot of Nigeria's daily COVID-19 death cases by gender from April 11, 2020 to September 12, 2020. The highest daily death cases were recorded on June 16, 2020.

\section{Dealing with missing values}

The data were characterized by eight missing values. The proportion of the original data values that were missing is 0.0516 for male and female daily incidence cases. For estimating missing values, multivariate imputation by chained equations [MICE] (van Buuren and GroothuisOudshoorn, 2011) based on random forest was implemented in this study. MICE was applied in Abiodun et al. (2019) to estimate a missing daily malaria count and missing values of some climate variables. MICE estimates missing values for continuous data using predictive mean matching approach and binary data using logistic regression (Abiodun et al., 2019). 
120

121

122

123

124

125

126

127

128

129

130

131

132

133

134

135

136

137

138

139

140

141

142

143

144

145

146

147

148

149

150

\section{Multivariate Ljung-Box test}

Multivariate Ljung-Box (MLB) test (Tsay, 2014) can be employed to investigate whether the daily incidence cases $\left(\boldsymbol{Z}_{t}\right)$ are autocorrelated or random. The MLB test is a multivariate generalisation of the univariate Ljung-Box test. The test rejects the null hypothesis that the daily incidence cases $\left(\boldsymbol{Z}_{t}\right)$ are random if $\mathrm{p}$-value of the test is less than a chosen level of significance.

\section{Augment Dickey-Fuller test}

Augmented Dickey-Fuller (ADF) test (Dickey and Fuller, 1979) is a statistical test for finding out if a time series contains a unit root. A time series has a unit root implies that the time series is not stationary. The null hypothesis for the ADF test is that a time series has a unit root. A nonstationary time series is differenced d times until it attains stationarity.

\section{Lagrange Multiplier test}

Lagrange Multiplier (LM) test (Tsay, 2014) was developed to investigate whether a vector time series contains conditional heteroscedasticity. The LM test can be employed to test for autoregressive conditional heteroscedasticity effects in the daily COVID-19 incidence. The test rejects the null hypothesis that there are autoregressive conditional heteroscedasticity effects in the daily COVID-19 incidence if p-value of the test is less than a chosen level of significance.

\section{Vector autoregressive moving average model of order $p$ and $q$}

Vector autoregressive moving average model of order $p$ and $q$, denoted by VARMA (p,q), is a multivariate generalization of univariate autoregressive moving average model for stationary time series. Mathematically, the $\operatorname{VARMA}(\mathrm{p}, \mathrm{q})$ model is defined as

$$
\boldsymbol{Z}_{t}=\boldsymbol{\mu}_{t}+\boldsymbol{\Phi}_{1} \boldsymbol{Z}_{t-1}+\boldsymbol{\Phi}_{2} \boldsymbol{Z}_{t-2}+\ldots+\boldsymbol{\Phi}_{p} \boldsymbol{Z}_{t-p}+\boldsymbol{\varepsilon}_{t}+\boldsymbol{\theta}_{1} \boldsymbol{\varepsilon}_{t-1}+\boldsymbol{\theta}_{2} \boldsymbol{\varepsilon}_{t-2}+\ldots+\boldsymbol{\theta}_{q} \boldsymbol{\varepsilon}_{t-q}
$$

where $\boldsymbol{Z}_{t}=\left(\begin{array}{c}M_{t} \\ F_{t}\end{array}\right)$ is a vector of male and female incidence at time $t, \boldsymbol{\mu}_{t}=\left(\begin{array}{l}\mu_{1, t} \\ \mu_{2, t}\end{array}\right)$ is an intercept vector, $\boldsymbol{\varepsilon}_{t}=\left(\begin{array}{l}\varepsilon_{1, t} \\ \varepsilon_{2, t}\end{array}\right)$ is a random error vector, $\boldsymbol{\Phi}_{i}=\left(\begin{array}{ll}\phi_{i, 11} & \phi_{i, 12} \\ \phi_{i, 21} & \phi_{i, 22}\end{array}\right), i=1,2, \ldots, p$ is a parameter of vector autoregressive part and $\boldsymbol{\theta}_{i}=\left(\begin{array}{ll}\theta_{i, 11} & \theta_{i, 12} \\ \theta_{i, 21} & \theta_{i, 22}\end{array}\right), i=1,2, \ldots, q$ is a parameter of vector moving average part. The random error vectors $\boldsymbol{\varepsilon}_{t}, \boldsymbol{\varepsilon}_{t-1}, \ldots, \boldsymbol{\varepsilon}_{t-q}$ are independently, identically, and normally distributed with mean zero and covariance matrix $\boldsymbol{\Sigma}=E\left(\boldsymbol{\varepsilon}_{t}^{\prime} \boldsymbol{\varepsilon}_{t}\right)=\left(\begin{array}{ll}\sigma_{11} & \sigma_{12} \\ \sigma_{21} & \sigma_{22}\end{array}\right)$. When time series $\left(\boldsymbol{Z}_{t}\right)$ in not stationary, the difference operator $\Delta^{d}$ is employed on $\boldsymbol{Z}_{t}$ to achieve stationarity. The difference operator $\Delta^{d}$ is defined as 


$$
\Delta^{d}(B)=\left(\begin{array}{cccc}
(1-\mathrm{B})^{\mathrm{d}_{1}} & 0 & \ldots & 0 \\
0 & (1-\mathrm{B})^{\mathrm{d}_{2}} & \cdots & 0 \\
\vdots & \vdots & \ddots & \vdots \\
0 & 0 & \ldots & (1-\mathrm{B})^{\mathrm{d}_{k}}
\end{array}\right) .
$$

152 The coefficients of the VARMA model are estimated using conditional maximum likelihood 153 approach (Tsay, 2014).

154

155

156

157

158

159

160

161

162

163

164

165

166

167

168

169

170

171

172

173

174

175

176

177

178

179

180

181

\section{The univariate autoregressive integrated moving average model}

The univariate autoregressive integrated moving average model, denoted byARIMA( $p, d, q)$, on a Nigeria daily reported death cases $\left(X_{t}\right)$ at time $t$ is defined as

$$
\begin{aligned}
& \Delta^{d} X_{t} \\
& =c+\phi_{1} \Delta^{d} X_{t-1}+\phi_{2} \Delta^{d} X_{t-2}+\ldots+\phi_{p} \Delta^{d} X_{t-p}+\epsilon_{t}+\theta_{1} \epsilon_{t-1}+\theta_{2} \epsilon_{t-2}+\ldots+\theta_{q} \epsilon_{t-q}
\end{aligned}
$$

where $p, d$ and $q$ are orders of autoregressive, differenced and moving average parts

respectively, $\epsilon_{t}$ is the residual of the estimated $Y_{t}$, which is assumed uncorrelated. $\Delta$ is the difference operator, $\phi_{1}, \phi_{1}, \ldots, \phi_{p}$ are the parameters of the autoregressive part of the model, $\theta_{1}$ , $\theta_{2}, \ldots, \theta_{q}$ are parameters of the moving average part of the model. The choice of optimal values of $p$ and $q$ are based on the $\operatorname{ARIMA}(p, d, q)$ model with the least Bayesian information criterion. The coefficients of the ARIMA model are estimated using maximum likelihood estimation.

\section{Case-fatality risks of COVID-19 and fold change}

The crude case-fatality risk (cCFR) (Lau et al., 2020) of COVID-19 infections at date $t$ is defined as the ratio of the total number of deaths on day $t$ to the total number of confirmed cases on day $t$. Lau et al. (2020) defined an cCFR-adjusted total cases of country A at date $t$ relative to country $\mathrm{B}$ as

$$
\operatorname{ATCC}(A)=\text { total reported cases }(A) \times \frac{\operatorname{cCFR}(A)}{\operatorname{cCFR}(B)}
$$

where $\operatorname{ATCC}(A)$ is the cCFR-adjusted total COVID-19 cases of country A at date t, $c C F R(A)$ and $\operatorname{cCFR}(B)$ are crude case-fatality risk of countries A and B respectively at time $\mathrm{t}$. The fold change at date $\mathrm{t}$ is defined as

$$
\text { fold change at date } t=\frac{\mathrm{CCFR}-\text { adjusted total cases at date } \mathrm{t}}{\text { total reported cases at date } t} .
$$

\section{Results}

There is a significantly increasing trend ( $p$-value $\left.<2.22 \times 10^{-16}\right)$ in daily male incidence from April 11, 2020 to June 18, 2020 while there is a significantly decreasing trend ( $p$-value $=1.9091 \times 10^{-12}$ ) in daily male incidence from June 19, 2020 to September 12, 2020. There is a significantly increasing trend $\left(p\right.$-value $\left.<2.22 \times 10^{-16}\right)$ in daily female incidence from 
182 April 11 to July 13, 2020 while there is a significantly decreasing trend ( $p$-value $=1.4916 \times$ $18310^{-6}$ ) in daily female incidence from July 14, 2020 to September 12, 2020. Similarly, there is a 184 significantly increasing trend ( $p$-value $<2.22 \times 10^{-16}$ ) in daily total incidence from April 11, 1852020 to July 1,2020 while there is a significantly decreasing trend ( $p$-value $=2.084 \times 10^{-12}$ ) in 186 daily male incidence from July 2, 2020 to September 12, 2020. There is a significantly increasing 187 trend $\left(p\right.$-value $=3.5763 \times 10^{-7}$ ) in daily death cases from April 11, 2020 to June 16, 2020

188

189

190

191

192

193

194

195

196

197

198

199

200

201

202

203

204

205

206

207

208

209

210

211

212

213

214

215

216

$217=0.9069)$. 218 The difference operation of the daily COVID-19 incidence was performed. The data was 219 while there is a significantly decreasing trend $\left(p\right.$-value $\left.=2.1484 \times 10^{-14}\right)$ in daily death cases from June 17, 2020 to September 12, 2020.

White-neural-network test shows that daily female incidence is not linear in mean ( $p$-value $=$ 0.00058746 ) while daily male incidence is linear in mean ( $\mathrm{p}$-value $=0.4257)$. McLeod-Li test was applied to investigate whether there are no autoregressive conditional heteroscedasticity effects in the daily incidence cases. There is an autoregressive conditional heteroscedasticity effect in daily female incidence if the mean daily female incidence changes over time. This implies that on the average, the daily female incidence changes significantly over time. This is shown in Figure 3. The McLeod-Li test shows that there is autoregressive conditional heteroscedasticity in the female incidence (Maximum $p$-value $=1.4277 \times 10^{-5}$ ) and male incidence (Maximum $p$ value $=9.0816 \times 10^{-14}$ ) at $5 \%$ level of significance. This is confirmed by the LM test for vector of male and female incidence. The LM test shows that there are autoregressive conditional heteroscedasticity effects in the daily COVID-19 incidence ( $p$-value $=0.0001)$. Similarly, the multivariate Ljung-Box test (Tsay, 2014) shows that the daily incidence cases $\left(\boldsymbol{Z}_{t}\right)$ are not random $(p$-value $=0.0000)$. This implies that the daily incidence cases are autocorrelated. Figure 4 presents the plot of cross correlation between the daily male and female incidence at $k$ previous days. It was observed that the cross correlation values are positive at all lags and significant up to 18 previous days. This implies that daily female incidence at day $t$ is significantly increasing as the daily male incidence increases at day $t-k$.

The Wilcoxon signed-rank test was employed to test if the null hypothesis that confirmed male and female cases have the same distribution against the alternative hypothesis that the distributions of male and confirmed female cases are not the same. Result of the test shows that confirmed cases in male and female have different distributions ( $p$-value $<2.22 \times 10^{-16}$ ) and the number of confirmed cases is significantly higher in males than in females.

Augment Dickey-Fuller test was employed on each of the series to investigate if the confirmed male and female incidence cases are stationary at 5\% level of significance. The test rejects the hypothesis that a time series is not stationary if $p$-value of the test is less than 0.05 . Table 1 presents the test statistics and p-values of the test for incidence by gender. The Nigeria's daily COVID-19 incidence is not stationary for male $(p$-value $=0.9328)$ and female patients ( $p$-value differenced once to attain stationarity, that is $\Delta^{d}(B)=\left(\begin{array}{cc}1-B & 0 \\ 0 & 1-B\end{array}\right)$. Then, $\operatorname{VARMA}(\mathrm{p}, \mathrm{q})$ 
220

221

222

223

224

225

226

227

228

229

230

231

232

233

234

235

236

237

238

239

240

241

242

243

244

245

246

247

248

249

250

251

252

253

254

255

256

257

\section{8}

model of various orders were fitted to the differenced data with the aim of identifying the optimal VARMA model for the data.

The optimal VARMA model is chosen as the model that minimises the Bayesian information criterion. The optimal model is VARMA $(0,1)$ model. Table 2 presents the estimates of coefficients of optimal VARMA $(0,1)$ model. The optimal VARMA $(0,1)$ model can be expressed mathematically as:

$$
\boldsymbol{Z}_{t}-\boldsymbol{Z}_{t-1}=\boldsymbol{\mu}_{t}+\boldsymbol{\varepsilon}_{t}+\boldsymbol{\theta}_{1} \boldsymbol{\varepsilon}_{t-1}
$$

where

$$
\boldsymbol{\mu}_{t}=\left(\begin{array}{c}
0.4836 \\
-0.4532
\end{array}\right), \quad \boldsymbol{\theta}_{1}=\left(\begin{array}{cc}
-0.953 & 0.348 \\
0.101 & -0.861
\end{array}\right),
$$

and the covariance matrix of residuals is $\boldsymbol{\Sigma}=\left(\begin{array}{cc}5166.7336 & -187.7683 \\ -187.7683 & 3378.1531\end{array}\right)$.

The optimal VARMA $(0,1)$ model can also be presented as two univariate regression models:

$$
\begin{aligned}
& M_{t}-M_{t-1}=0.484+\varepsilon_{t}-0.953 \varepsilon_{t-1} \\
& F_{t}-F_{t-1}=-0.453+\varepsilon_{t}-0.861 \varepsilon_{t-1}
\end{aligned}
$$

The test of significance of estimates of coefficients of the models shows that estimates of coefficients of the models are significantly different from zero (p-value $<0.05$ ). This is given in Table 2. The implication of the above equations is that daily male incidence does not significantly depend on female incidence at previous days. Similarly, number of female incidence does not depend on male incidence at previous days. Figure 5 presents the comparison of the observed incidence with fitted data by gender. It can be observed from the figure that the models fit the data well.

The calculated cCFR value of Nigeria is 0.0192 . The total reported cases as of September 12, 2020 is 440248 . The calculated cCFR value of Germany as of September 12, 2020 is 0.0362 .

The total reported cases as of September 12, 2020 is 440248 while the estimated total COVID-19 incidence cases based on cCFR is 233,188 . The fold change value of Nigeria is estimated to be 0.5297 .

\section{Modelling Nigeria's daily COVID-19 death cases}

Augmented Dickey-Fuller (ADF) test was employed to investigate the stationarity of Nigeria's daily COVID-19 death cases. The ADF test (p-value $=0.0888$ ) fails to reject the null hypothesis that the daily reported death cases are stationary at $5 \%$ level of significance. This implies that the daily COVID-19 death cases are not stationary. That is, the average and variance of reported death cases change with time. To overcome this, a univariate autoregressive moving average (ARIMA) model of various orders was formulated for Nigeria's daily COVID-19 death cases. Optimal model was selected following Makinde and Fasoranbaku (2011) and Benvenuto et al. (2020). The optimal model for the Nigeria's daily COVID-19 death cases is identified to be $\operatorname{ARIMA}(0,1,1)$. Figure 6 presents the comparison of the observed death cases with fitted data. It can be observed from the figure that the optimal ARIMA $(0,1,1)$ model fits the data well.

\section{Discussion}


259 Daily confirmed cases in Nigeria are gender-skewed (Figure 1). The daily male-female ratios of 260 COVID-19 confirmed cases are higher than one over the study period. This is confirmed in 261 Figure 3. In the figure, daily average confirmed cases are consistently higher in males but 262 monotonically increasing till August 8, 2020. Result of Wilcoxon signed-rank test shows that 263 confirmed male cases are not distributed equivalently as confirmed female cases. The result of 264 the test confirms that confirmed male and female cases have different distributions and the 265 number of confirmed cases is significantly higher in males. Bianconi et al. (2020) argued that 266 gender inequalities may have influenced either the estimation of COVID-19 cases or the 267 susceptibility to COVID-19 over time. Bianconi et al. (2020) observed a time-related changes in 268 sex distribution of COVID-19 cases in Italy as well as declining infection proportion with male269 female ratio from March 9 to May 11, 2020. However, there is no significant time-related 270 changes in sex distribution of COVID-19 cases in Nigeria. Bianconi et al . (2020) argued that 271 males have been reported to be at higher risk of developing symptoms and severe clinical 272 manifestations of COVID-19, and may have had a higher access to COVID-19 diagnostic tests at 273 the beginning of the observed period as compared to females.

274 Gebhard et al. (2020) claimed that COVID-19 is deadlier for infected men than women in China 275 in terms of fatality rate, and that differential susceptibility between males and females may be 276 imparted in diverse ways by gender for infectious diseases caused by viruses. Klein and Huber 277 (2009) argued that susceptibility and response to viral infections, incidence and disease severity 278 may differ in males and females. Gadi et al. (2020) attributed higher risks of COVID-19 in males 279 to the lack of the stimulatory effects of estrogen and that androgens which males produce seem 280 to have a defensive mechanism against their immune response. Jin et al. (2020) investigated the 281 role of gender in morbidity and mortality in patients with COVID-19 in China and concluded 282 that men could be more at risk of death by COVID-19.

283 The confirmed male and female incidence cases were not stationary. This implies that either their

284

285

286

287

288

289

290

291

292

293

294

295

296

297

298 means, variances or covariances increased with time (Figure 3). The confirmed male and female incidence cases were differenced once in order to achieve stationarity. The VARMA model was fitted to the differenced data. The fitted VARMA model showed that daily male incidence does not significantly depend on female incidence at previous days. Similarly, number of female incidence does not depend on male incidence at previous days. Comparison of the observed incidence with fitted data showed that the models fit the data well (Figure 5). Similarly, ARIMA $(0,1,1)$ model was selected as the optimal ARIMA $(p, d, q)$ for fitting daily reported death cases using Bayesian information criterion. The model fits the data well, as shown in Figure 6. This is in line with study of Benvenuto et al. (2020), where ARIMA models were performed on the Johns Hopkins epidemiological data to predict the epidemiological trend of the prevalence and incidence of COVID-19. However, parameters of optimal ARIMA models were not identified in their study.

In evaluating massive under-reporting and under-testing of total reported COVID-19 cases in some global epicenters, cCFR value of Germany was used as a standard in Lau et al. (2020). In this study, cCFR value of Germany was also considered as the standard. As of September 12, 
299

300

301

302

303

304

305

306

307

308

309

310

311

312

313

314

315

316

317

318

319

320

321

322

323

324

325

326

327

328

329

330

331

332

333

334

335

336

337

338

2020, the cCFR values of Germany, Greece and South Korea are 3.62\%, 2.36\% and 1.61\% respectively while the cCFR value of Nigeria is $1.92 \%$. The estimated total COVID-19 incidence cases is 233,188 . There is no evidence of massive under-detection and under-reporting in Nigeria. When estimating the real total amount of COVID-19 cases in Nigeria, no considerable increase in the number of COVID-19 incidence is detected in comparison to Nigeria's total reported COVID-19 cases. Fold change can be used as an indicator for under-reporting and under-detecting COVID-19 cases. Nigeria's fold change value is estimated to be 0.5297 as of September 12, 2020. This value is comparable to fold change of South Korea but less than that of Italy, France, Spain, Iran, China and the United States of America as of March 17, 2020. This study is limited by dearth of information on mortality cases by gender and daily population in Nigeria.

\section{Conclusions}

This study provides insight into how COVID-19 affects one sex than the other in Nigeria over a period of time. In particular, a vector autoregressive moving average model was formulated for predicting gender based - daily incidence in Nigeria. Also, a predictive model based on autoregressive integrated moving average model was presented for daily death cases. The findings show that males were at higher risk of contracting COVID-19 than females. The mean daily incidence changes over time for male and female but the change is significant for female individuals. The significance of gender on daily incidence was highlighted. The vector autoregressive moving average model shows that male incidence does not depend on number of female incidence at previous days. Similarly, number of female incidence does not depend on number of male incidence at previous days. Massive underreporting and under-testing was not observed in Nigeria's reported COVID-19 cases based on crude case-fatality risk and fold change.

\section{References}

Abdulmajeed, K., Adeleke, M. and Popoola, L. (2020). Online forecasting of COVID-19 cases in Nigeria using limited data. Data in Brief, 30:105683. doi:10.1016/j.dib.2020.105683

Abiodun, G.J., Makinde, O.S., Adeola, A.M., Njabo, K.Y., Witbooi, P.J., Djidjou-Demasse, R. and Botai, J.O. (2019) A dynamical and zero-inflated negative binomial regression modelling of malaria incidence in Limpopo Province, South Africa. International Journal of Environmental Research and Public Health, 16(11):s2000. doi:10.3390/ijerph16112000

Benvenuto, D., Giovanetti, M., Vassallo, L., Angeletti, S., Ciccozzi, M. (2020) Application of the ARIMA model on the COVID-2019 epidemic dataset. Data in Brief, 29:105340

Bianconi V., Mannarino M.R., Bronzo P., Marini E., Pirro M. (2020) Time-related changes in sex distribution of COVID-19 incidence proportion in Italy. Heliyon, 6:e05304

Bowale, A., Abayomi, A., Idris, J., Omilabu, S., Abdus-Salam, I., Adebayo, B., Opawoye, F., Finnih-Awokoya, O., Zamba, E., Abdur-Razzaq, H., Erinoso, O., Onasanya, T., Ramadan, P., Nyenyi, Z., Aniaku, E., Balogun, M., Okunromade, O., Adejumo, O., Adesola, S., 
Ogunniyan, T., Balogun, M. and Osibogun, A. (2020). Clinical presentation, case management and outcomes for the first 32 COVID-19 patients in Nigeria, Pan Africa Medical Journal. 35(2):24. doi: 10.11604/pamj.2020.35.2.23262

Cascella, M., Rajnik, M., Cuomo, A., Dulebohn, S.C. and Di Napoli R. (2020). Features, evaluation and treatment coronavirus (COVID-19). StatPearls Publishing.

Del Rio, C. and Malani, P.N. (2020). COVID-19-new insights on a rapidly changing epidemic. Journal of the American Medical Association, 323(14):1339-1340.

Dickey, D.A. and Fuller, W.A. (1979). Distribution of the estimators for autoregressive time series with a unit root. Journal of the American Statistical Association, 74(366), 427-431. doi: $10.2307 / 2286348$

Drosten, C., Gunther, S., Preiser, W., van der Werf, S., Brodt, H.R., Becker, S., Rabenau, H., Panning, M., Kolesnikova, L., Fouchier, R., Berger, A., Burguière, A., Cinatl, J., Eickmann, M., Escriou, N., Grywna, K., Kramme, S., Manuguerra, J., Müller, S., Rickerts, V., Stürmer, M., Vieth, S., Klenk, H., Osterhaus, A., Schmitz, H. and Doerr, H. (2003). Identification of a novel coronavirus in patients with severe acute respiratorysyndrome. New England Journal of Medicine, 348:1967-76. doi: 10.1056/NEJMoa030747

Gadi, N., Wu, S.C., Spihlman, A.P. and Moulton, V.R. (2020) What's sex got to do with covid19? Gender-based differences in the host immune response to coronaviruses. Frontiers in Immunology, doi: 10.3389/fimmu.2020.02147

Gebhard C, Regitz-Zagrosek V, Neuhauser HK, Morgan R and Klein SL. (2020) Impact of sex and gender on COVID-19 outcomes in Europe. Biology of Sex Differences, 11:29 https://doi.org/10.1186/s13293-020-00304-9

Gorbalenya, A.E., Baker, S.C., Baric, R.S., de Groot, R., Drosten, C., Gulyaeva, A.A., Haagmans, B., Lauber, C., Leontovich, A.M., Neuman, B.W., Penzar, D., Perlman, S., Poon, L.M., Samborskiy, D.V., Sidorov, I.A., Sola, I. and Ziebuh, J. (2020). The species Severe acute respiratory syndrome-related coronavirus: classifying 2019-nCoV and naming it SARS-CoV-2. Nature Microbiology, 5(4):536-544.

Jin, J-M., Bai, P., He, W., Wu, F., Liu, X-F., Han, D-M., Liu, S., Yang, J-K. (2020) Gender Differences in Patients With COVID-19: Focus on Severity and Mortality. Frontiers in Public Health, 8:152. doi: 10.3389/fpubh.2020.00152

Klein, S.L. and Huber, S. (2009) Sex differences in susceptibility to viral infection. In: Klein SL, Roberts CW, editors. Sex Hormones and Immunity to Infection. Berlin: Springer-Verlag.

Lau H., Khosrawipour T., Kocbach P., Ichii H., Bania J., Khosrawipour V. (020) Evaluating the massive underreporting and undertesting of COVID-19 cases in multiple global epicenters,Pulmonology, 1502. https://doi.org/10.1016/j.pulmoe.2020.05.015

Makinde, O.S. and Fasoranbaku, O.A. (2011). Identification of Optimal Autoregressive Integrated Moving Average Model on Temperature Data. Journal of Modern Applied Statistical Methods, 10(2):718-729. 
377

378

379

380

381

382

383

384

385

386

387

388

389

390

391

392

393

394

395

396

397

398

399

400

401

402

403

404

405

406

407

408

409

410

Makinde, O.S., Olusola-Makinde, O.O., Olamide E.I. and Abiodun, G.J. (2020a). Impact of COVID-19 prevalence and mode of transmission on mortality cases over WHO regions. Health Information Science and System, 8:35

Makinde, O.S., Oseni, B.M., Adepetun, A.O., Olusola-Makinde, O.O. and Abiodun, G.J. (2020b). The significance of daily incidence and mortality cases due to covid-19 in some African countries. In Data Science for COVID-19 (Kose et al. (eds)). Elsevier (To appear).

Nigeria Centre for Disease Control (NCDC) (2020a). First Case of Coronavirus Disease Confirmed in Nigeria 2020a [cited September 6, 2020]. Available from:

https://ncdc.gov.ng/news/227/first-case-of-corona-virus-disease-confirmed-in-nigeria.

Nigeria Centre for Disease Control (NCDC) (2020b). COVID-19 Outbreak In Nigeria Situation Report S/N 54. Abuja. Available from: https://ncdc.gov.ng/diseases/sitreps/?cat=14\&name=An\%20update\%20of\%20COVID19\%20outbreak\%20in\%20Nigeria [accessed September 6, 2020].

Ojokoh, B.A., Makinde, O.S., Fayeun, L.S., Babalola, O.T., Salako, K.V. and Adzitey, F. (2020) Impact of COVID-19 and lockdown policies on farming, food security and agribusiness in West Africa. In Data Science for COVID-19 (Kose et al. (eds)). Elsevier (To appear).

Purcell, L.N. and Charles, A.G. (2020). An invited commentary on World Health Organization declares global emergency: a review of the 2019 novel Coronavirus (COVID-19): emergency or new reality. International Journal of Surgery, 76:111.

Sohrabi, C., Alsafi, Z., O’Neill, N., Khan, M., Kerwan, A., Al-Jabir, A., Iosifidis, C. and Agha, R. (2020). World Health Organization declares global emergency: a review of the 2019 Novel Coronavirus (COVID-19). International Journal of Surgery, 76:71-76.

Tsay, R.S. (2014). Multivariate Time Series Analysis with R and Financial Applications. John Wiley. Hoboken, NJ

UNWOMEN. (2020). Emerging Gender Data and Why It Matters, Available from: https://data.unwomen.org/resources/covid-19-emerging-gender-data-and-why-it-matters, (accessed September 6, 2020)

van Buuren, S. and Groothuis-Oudshoorn, K. (2011). Multivariate Imputation by Chained Equations in R. Journal of Statistical Software, 45:1-67.

Zhu N, Zhang D, Wang W, Li X, Yang B, Song J, Zhao, X., Huang, B., Shi, W., Lu, R., Niu, P., Zhan, F., Ma, X., Wang, D., Xu, W., Wu, G., Gao, G., Tan, W. and China Novel Coronavirus Investigating and Research Team. (2020). A novel coronavirus from patients with Pneumonia in China, New England Journal of Medicine, 382:727-33. doi: 10.1056/NEJMoa2001017. 
Figure 1

Comparison of observed data with fitted confirmed incidence

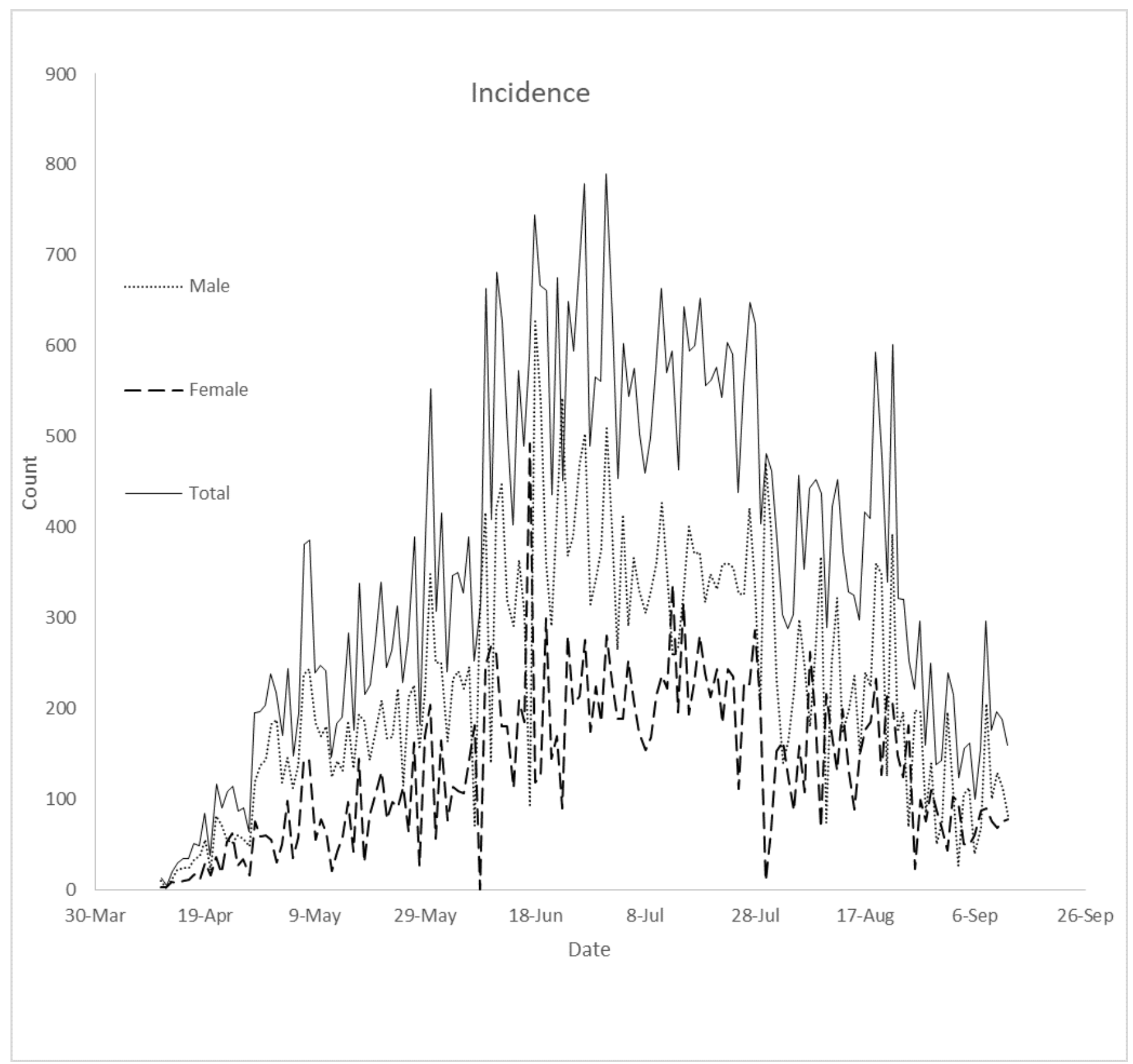


Figure 2

Plot of number of Nigeria's daily COVID-19 death cases from April 11, 2020 to September 12, 2020 


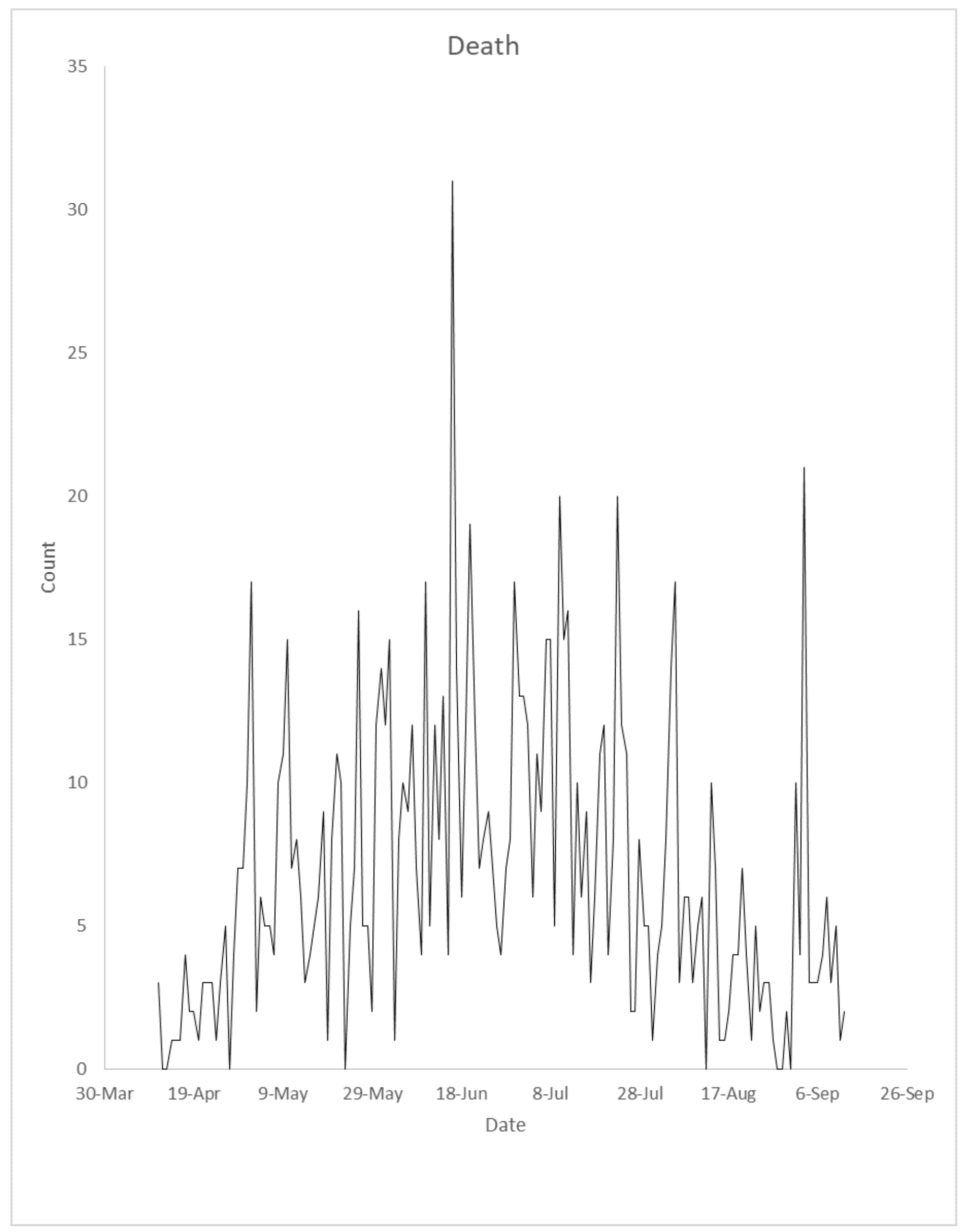


Figure 3

Plot of daily mean incidence over time 


\section{Mean incidence over time}

450

400

Male incidence

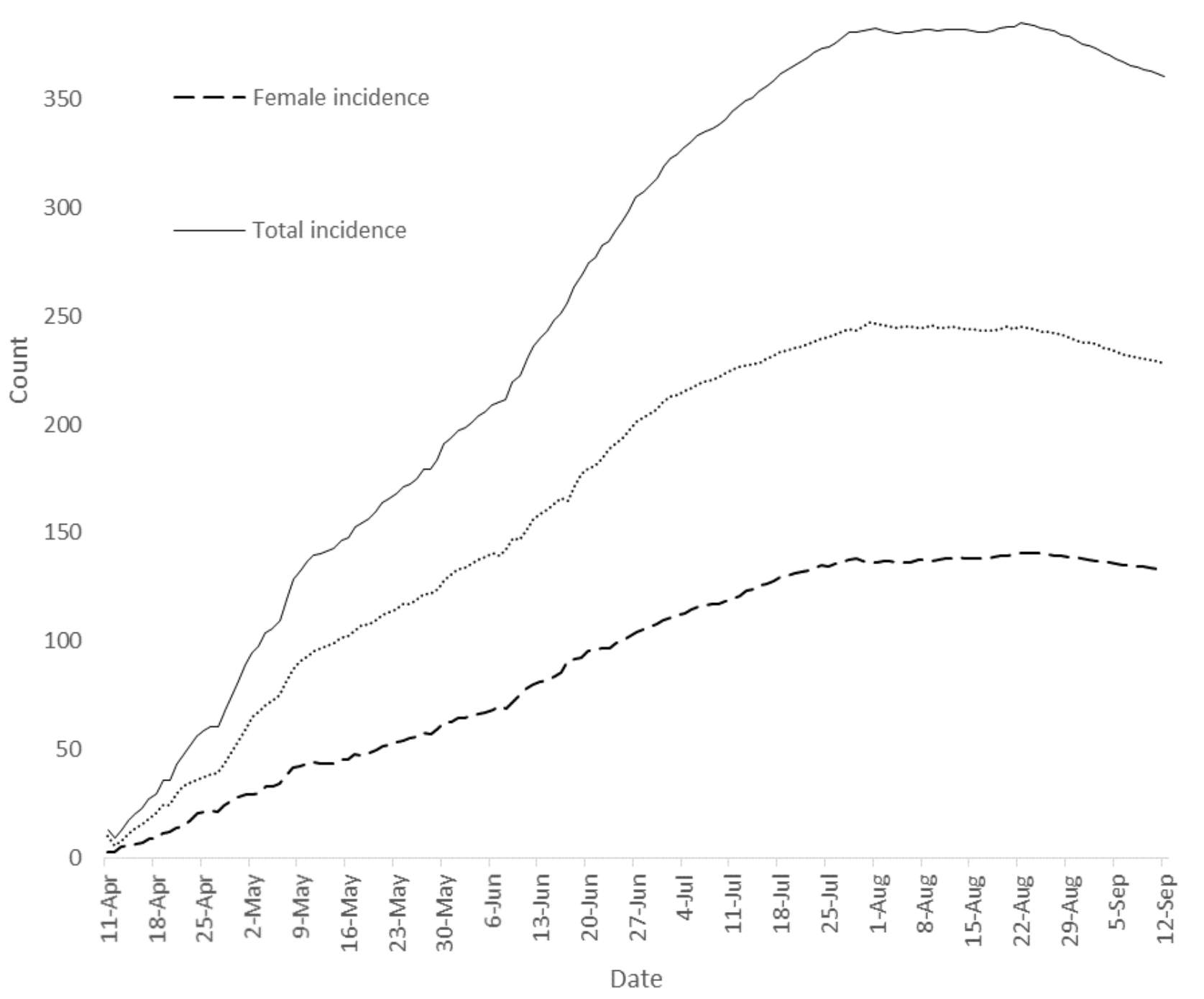


Figure 4

Plot of cross correlation function for daily male and female COVID-19 incidence in Nigeria

\section{Male \& Female}

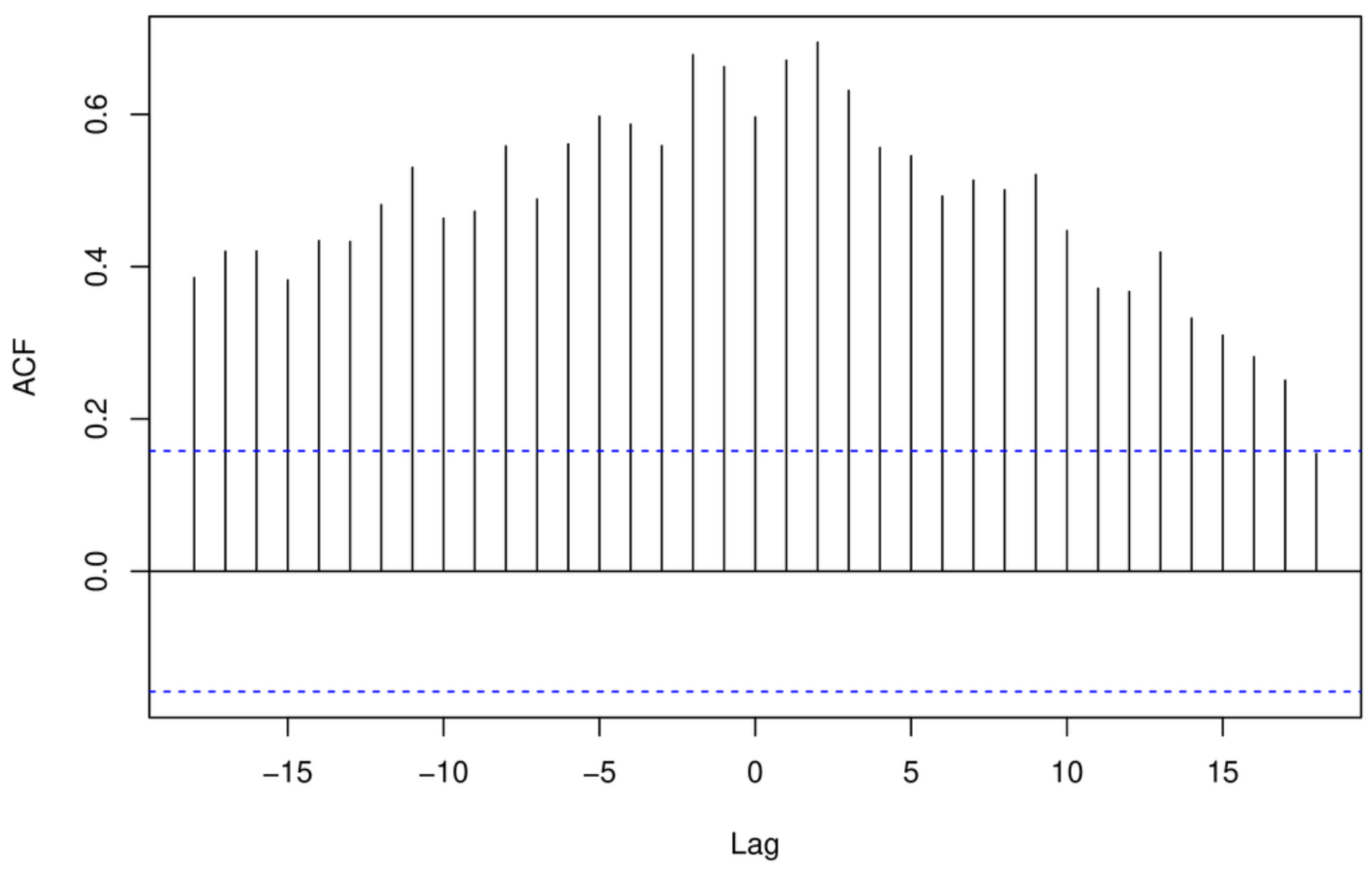


Figure 5

Comparison of observed data with fitted confirmed incidence 


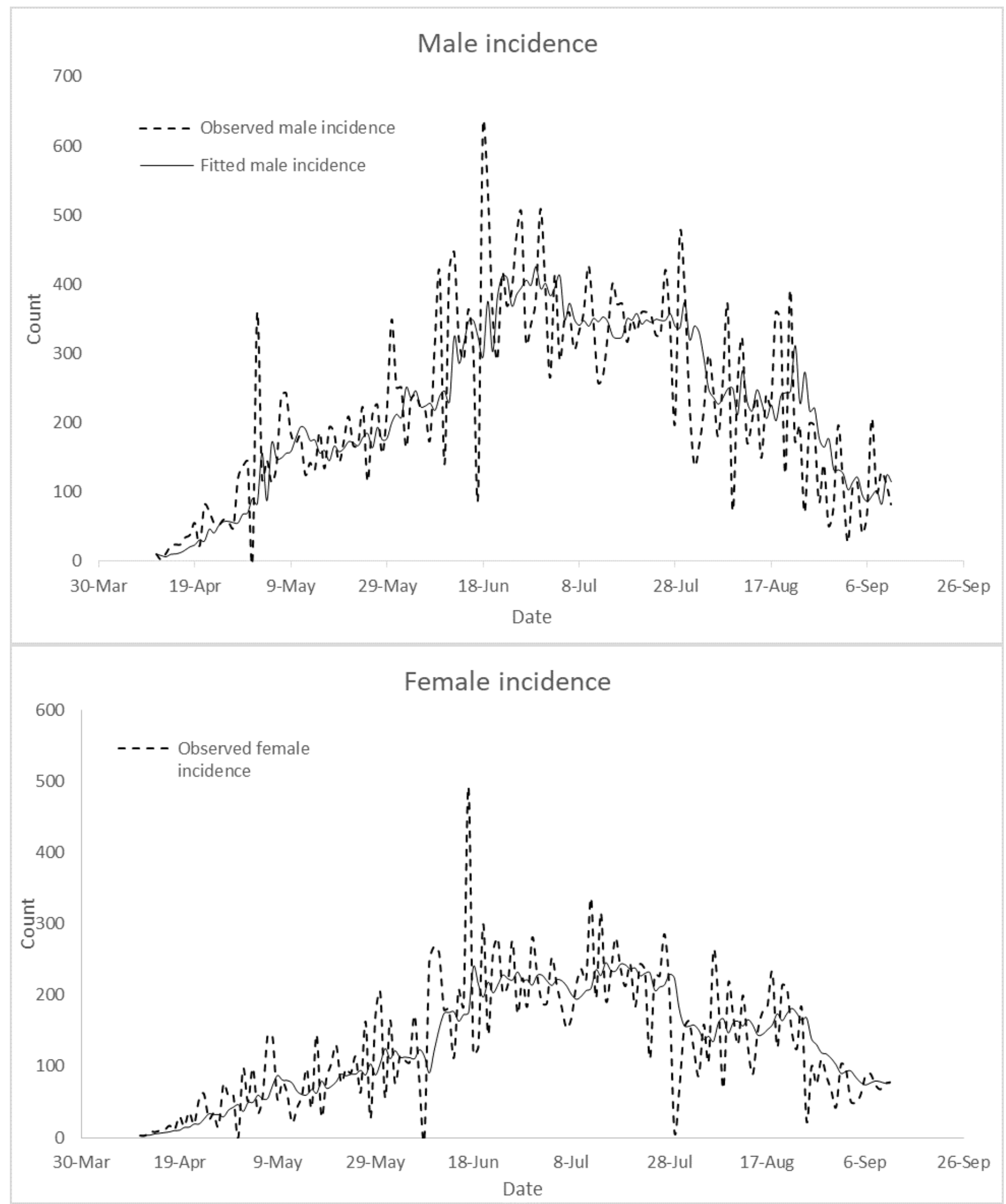


Figure 6

Comparison of observed data with fitted death cases in Nigeria 


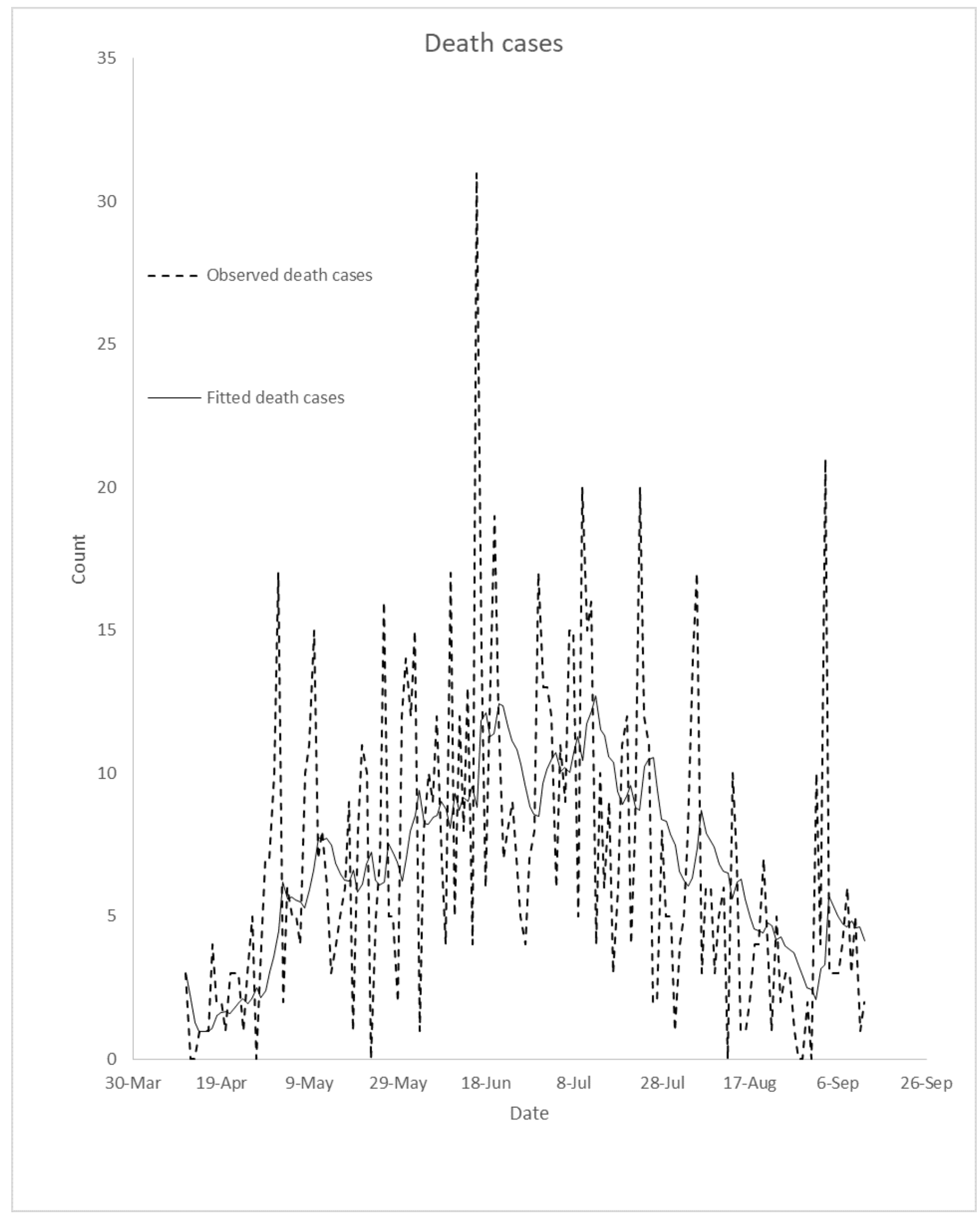




\section{Table $\mathbf{1}$ (on next page)}

Augmented Dickey-Fuller test of stationarity for Nigeria's daily COVID-19 incidence 
1

\begin{tabular}{lcc}
\hline & Male & Female \\
\hline Dickey-Fuller test statistic & -1.3750 & -1.4331 \\
p-value & 0.8369 & 0.8127 \\
\hline
\end{tabular}

2

3 
Table 2 (on next page)

Estimates of coefficients of optimal VARMA $(0,1)$ models 
1

\begin{tabular}{ccccc}
\hline Coefficients & Estimate & Std. Error & t value & $\operatorname{Pr}(>|\mathrm{t}|)$ \\
\hline$\mu_{1, t}$ & 0.484 & $9.24 \times 10^{-6}$ & 52353 & $<2 \times 10^{-16 * * *}$ \\
$\mu_{2, t}$ & -0.453 & $8.61 \times 10^{-6}$ & -52670 & $<2 \times 10^{-16 * * *}$ \\
$\theta_{1,11}$ & -0.953 & $5.91 \times 10^{-6}$ & -161193 & $<2 \times 10^{-16 * * *}$ \\
$\theta_{1,12}$ & 0.348 & $2.36 \times 10^{-6}$ & 147627 & $<2 \times 10^{-16 * * *}$ \\
$\theta_{1,21}$ & 0.101 & $9.44 \times 10^{-7}$ & 106724 & $<2 \times 10^{-16 * * *}$ \\
$\theta_{1,22}$ & -0.861 & $5.08 \times 10^{-6}$ & -169620 & $<2 \times 10^{-16 * * *}$ \\
\hline
\end{tabular}

2 